\title{
Stabilization with Disturbance Attenuation over a Gaussian Channel
}

\author{
J. S. Freudenberg, R. H. Middleton, and J. H. Braslavsky
}

\begin{abstract}
We propose a linear control and communication scheme for the purposes of stabilization and disturbance attenuation when a discrete Gaussian channel is present in the feedback loop. Specifically, the channel input is amplified by a constant gain before transmission and the channel output is processed through a linear time invariant filter to produce the control signal. We show how the gain and filter may be chosen to minimize the variance of the plant output. For an order one plant, our scheme achieves the theoretical minimum taken over a much broader class of compensators.
\end{abstract}

\section{INTRODUCTION}

Many authors have studied the problem of controlling a linear system with a communication channel in the feedback loop (e.g., [1]). The most general framework for doing so allows compensation at the channel input and output that may be nonlinear, time-varying, and dynamical. In the present paper we shall consider the simpler communication and control scheme shown in Figure 1 wherein the channel precompensator is assumed to be a constant gain, $\lambda$, and the postcompensator is assumed to be a causal linear time-invariant filter. The plant is discrete, linear, and timeinvariant, and the channel is Gaussian with input power limit $P$ and noise variance $\sigma_{n}^{2}$. The purpose of control is to stabilize the plant, if necessary, and to minimize the variance of the plant output in response to a Gaussian disturbance. It is the simple nature of the communication and control scheme in Figure 1 that motivates us to study its properties.

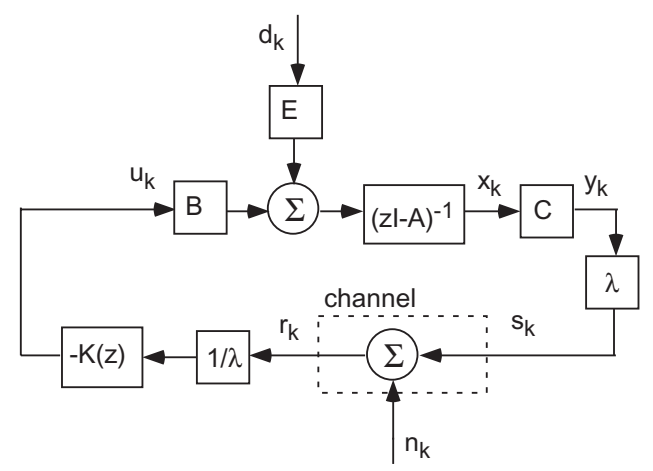

Fig. 1. Feedback control over a Gaussian communication channel with input power constraint $\mathcal{E}\left\{s_{k}^{2}\right\}<P$ and additive white noise of variance $\sigma_{n}^{2}$

J. S. Freudenberg is with Department of Electrical Engineering and Computer Science, The University of Michigan, Ann Arbor, MI, 48109-2122, USA jfr@eecs.umich.edu. R H. Middleton is with the Hamilton Institute, National University of Ireland Maynooth, Co Kildare, Ireland. richard.middleton@nuim.ie. J. H. Braslavsky is with the Centre for Complex Dynamic Systems and Control, The University of Newcastle, 2308, Australia julio.braslavsky@newcastle.edu.au.
If the scalar amplifier at the channel input were a unity gain, and if the power constraint were not present, then the problem of minimizing the variance of the plant output would be a standard linear quadratic Gaussian (LQG) control problem, whose solution is state feedback applied to a state estimate obtained from a Kalman filter. We show that the LQG results may be modified to apply with the pre-channel amplification and the power limit present.

The remainder of this paper is outlined as follows. In Section II we provide a precise problem statement and develop necessary background. Standard LQG results are applied in Section III for the case $\lambda=1$. We show that, under appropriate hypotheses, the minimal output variance is equal to that of the minimal estimation error for the predicting version of the optimal estimator. In Section IV we use the concept of entropy rate to develop a formula for the optimal estimation error and the minimal channel capacity. In Section $\mathrm{V}$ this formula is modified to include nonunity values of $\lambda$, and we show that the variance of the plant output is minimized by choosing $\lambda$ so that the channel input satisfies the power limit arbitrarily closely. An example is also given in Section V. In Section VI, we show that, for a first order plant, our linear communication and control scheme achieves a theoretical lower bound on disturbance response that holds for general nonlinear, time-varying, and dynamical control and communication schemes. Conclusions and directions for further research are presented in Section VII.

\section{PRELIMINARIES}

Denote a random sequence by $x=\left\{x_{k}\right\}$, and define the subsequence $x^{k} \triangleq\left\{x_{\ell} ; \ell \leq k\right\}$. Unless stated otherwise, all signals are assumed to have stationary distributions. Hence if $x$ is a scalar valued sequence, the variance of $x$ is given by $\sigma_{x}^{2}=\mathcal{E}\left\{x_{k}^{2}\right\}$, and may be computed from its power spectral density $\mathcal{S}_{x}(\omega)$ by $\sigma_{x}^{2}=(1 / 2 \pi) \int_{-\pi}^{\pi} \mathcal{S}_{x}(\omega) d \omega$, if the spectral density is well-defined. The open and closed unit disks are denoted by $\mathbb{D}$ and $\overline{\mathbb{D}}$. A rational transfer function $G(z)$ is minimum phase if all its zeros lie in $\overline{\mathbb{D}}$, and is nonminimum phase (NMP) otherwise. We say that $G(z) \in H_{2}$ if $G(z)$ is strictly proper and all its poles lie in $\mathbb{D}$. The $H_{2}$ norm of $G(z) \in H_{2}$ is given by $\|G\|_{H_{2}}^{2}=(1 / 2 \pi) \int_{-\pi}^{\pi}\left|G\left(e^{j \omega}\right)\right|^{2} d \omega$.

\section{A. Problem Statement}

We consider the feedback system of Figure 1, with plant

$$
\begin{aligned}
x_{k+1} & =A x_{k}+B u_{k}+E d_{k}, x_{k} \in \mathbb{R}^{n}, u_{k}, d_{k} \in \mathbb{R}, \\
y_{k} & =C x_{k}, \quad y_{k} \in \mathbb{R},
\end{aligned}
$$

where $(A, B)$ and $(A, E)$ are assumed controllable, $(A, C)$ is assumed observable, and $d$ is a zero mean Gaussian white 
noise sequence of variance $\sigma_{d}^{2}$. The transfer functions from control and disturbance inputs to the plant output are denoted by $G_{u}=C \Phi B$ and $G_{d}=C \Phi E$, respectively, where $\Phi(z) \triangleq$ $(z I-A)^{-1}$. The output of the plant is multiplied by a scalar gain $\lambda$ and transmitted over a communication channel whose input $s$ and output $r$ are related by

$$
r_{k}=s_{k}+n_{k}
$$

where $n$ is a zero mean Gaussian white noise sequence of variance $\sigma_{n}^{2}$. The channel input $s$ is required to satisfy the power limit $\mathcal{E}\left\{s_{k}^{2}\right\}<P$, and thus the capacity of the channel is determined by the signal to noise ratio (SNR) $P / \sigma_{n}^{2}$ [2]: $\mathcal{C}=\frac{1}{2} \log _{e}\left(1+P / \sigma_{n}^{2}\right)$ nats/transmission. The channel output is scaled by $1 / \lambda$ and used as the input to a linear time invariant filter with transfer function $K(z)$, whose output is the control signal.

The goal of feedback control is to stabilize the plant, if necessary, and to attenuate the response of the plant output $y$ to the disturbance input $d$. Specifically, we seek $\lambda$ and $K(z)$ to minimize a cost function equal to the variance of the plant output

$$
J_{y} \triangleq \mathcal{E}\left\{y_{k}^{2}\right\}
$$

under the assumptions that the feedback system is internally stable, the channel power limit $P$ is satisfied, and the controller is causal. Denote the optimal value of the cost function (4) by

$$
J_{y}^{*} \triangleq \min _{K, \lambda} \mathcal{E}\left\{y_{k}^{2}\right\} .
$$

We shall also consider the problem of minimizing $\mathcal{E}\left\{y_{k}^{2}\right\}$ for a fixed value of $\lambda$, and denote the optimal cost by

$$
\left.J_{y}^{*}(\lambda) \triangleq \min _{K} \mathcal{E}\left\{y_{k}^{2}\right\}\right|_{\lambda} .
$$

For a fixed value of $\lambda$, the problem of choosing a causal controller to minimize (6) is a cheap control LQG optimization problem. We now provide a frequency domain version of the cost function. Under the assumption that all signal distributions are stationary, the variance of the system output $y$ may be computed from its power spectral density $\mathcal{S}_{y}(\omega)$, given by $\mathcal{S}_{y}(\omega)=\left|S\left(e^{j \omega}\right)\right|^{2}\left|G_{d}\left(e^{j \omega}\right)\right|^{2} \sigma_{d}^{2}+\left|T\left(e^{j \omega}\right)\right|^{2} \sigma_{n}^{2} / \lambda^{2}$, where $S$ and $T$ are the sensitivity and complementary sensitivity functions $S \triangleq 1 /\left(1+\lambda G_{u} K\right), T \triangleq 1-S$. It follows that $J_{y}=\left\|S G_{d}\right\|_{H_{2}}^{2} \sigma_{d}^{2}+\|T\|_{H_{2}}^{2} \sigma_{n}^{2} / \lambda^{2}$.

\section{B. SNR Limited Stabilization}

The authors of [3] consider the feedback system in Figure 1 with an unstable plant but without a plant disturbance, and determine the minimum value of $P$ required to stabilize the plant. With no disturbance present, there is no loss of generality in assuming $\lambda=1$, and the problem of minimizing (4) for a fixed noise variance is equivalent to that of minimizing the $\mathrm{H}_{2}$ norm of $T$.

Proposition II.1 ( [3] ) Consider the feedback system in Figure 1 with no disturbance and $\lambda=1$. Assume that $G_{u}$ has no nonminimum phase zeros and has relative degree equal to one. Suppose further that $G_{u}$ has poles $\phi_{i}, i=1, \ldots, m$, with $\left|\phi_{i}\right|>1$. Then there exists a controller $K(z)$ that stabilizes the feedback system if and only if the channel power constraint $P$ satisfies the lower bound $P>J_{y}^{*}(1)$, where $J_{y}^{*}(1)=\left(\prod_{i=1}^{m}\left|\phi_{i}\right|^{2}-1\right) \sigma_{n}^{2}$. Furthermore, the channel capacity required for stabilization with a linear time invariant controller must satisfy $\mathcal{C}>\sum_{i=1}^{m} \log _{e}\left|\phi_{i}\right|$ nats/transmission.

The authors of [4] show that if the plant is minimum phase and has relative degree one, then nonlinear, timevarying control and communication strategies cannot achieve stabilization with a channel capacity lower than that given in Proposition II.1 for linear control.

\section{The Discrete Time LQG Control Problem}

Consider the feedback system of Figure 1, and assume that $\lambda=1$. Under the assumption that all signals are stationary, the "cheap control" LQG cost function is given by $J_{L Q G}=$ $\mathcal{E}\left\{y_{k}^{2}\right\}$. It is well known [5] that the problem of finding a control law to stabilize the system and to minimize $J_{L Q G}$ has a solution given by state feedback applied to a state estimate obtained from an optimal estimator that is driven by the channel output. There are two possibilities for such an estimator, depending on whether or not the state estimate is allowed to depend on the current value of the channel output. We now review both versions of the estimator, as each plays a role in subsequent developments.

Consider first the state estimate of a predicting estimator, denoted $\hat{x}_{k \mid k-1}$, which depends only on previous values of the channel output. This estimate satisfies the state equations

$$
\hat{x}_{k+1 \mid k}=A \hat{x}_{k \mid k-1}+B u_{k}+L_{p}\left(r_{k}-C \hat{x}_{k \mid k-1}\right)
$$

where $L_{p}=A L_{f}, L_{f}=\Sigma C^{T}\left(C \Sigma C^{T}+\sigma_{n}^{2}\right)^{-1}$, and $\Sigma$ is the stabilizing solution to the Riccati equation

$$
\Sigma=A \Sigma A^{T}-A \Sigma C^{T}\left(C \Sigma C^{T}+\sigma_{n}^{2}\right)^{-1} C \Sigma A^{T}+\sigma_{d}^{2} E E^{T} .
$$

Define the output estimate and estimation error by $\hat{y}_{k \mid k-1}=$ $C \hat{x}_{k \mid k-1}$ and $\tilde{y}_{k \mid k-1}=y_{k}-\hat{y}_{k \mid k-1}$, respectively. Then the variance of the optimal predicting estimation error is given by

$$
\mathcal{E}^{*}\left\{\tilde{y}_{k \mid k-1}^{2}\right\}=C \Sigma C^{T} .
$$

The state estimate of the filtering version of the optimal estimator, denoted $\hat{x}_{k \mid k}$, does depend on the current value of the channel output, and satisfies

$$
\hat{x}_{k \mid k}=\hat{x}_{k \mid k-1}+L_{f}\left(r_{k}-C \hat{x}_{k \mid k-1}\right),
$$

where $\hat{x}_{k \mid k-1}$ is given by (7). The output estimate and estimation error are given by $\hat{y}_{k \mid k}=C \hat{x}_{k \mid k}$ and $\tilde{y}_{k \mid k}=$ $y_{k}-C \hat{x}_{k \mid k}$. The variance of the optimal filtering estimation error is equal to

$$
\mathcal{E}^{*}\left\{\tilde{y}_{k \mid k}^{2}\right\}=\frac{\sigma_{n}^{2} C \Sigma C^{T}}{\sigma_{n}^{2}+C \Sigma C^{T}} .
$$

If the filtering estimator is used to minimize $J_{L Q G}$, then the control law has the form

$$
u_{k}=-K_{c} \hat{x}_{k \mid k}
$$


where $\hat{x}_{k \mid k}$ is given by (10) and $K_{c}$ is found by solving a Riccati equation. The transfer function of the resulting compensator is given by

$$
K(z)=z K_{c}\left(z I-\left(I-L_{f} C\right)\left(A-B K_{c}\right)\right)^{-1} L_{p}
$$

A block diagram of the resulting feedback system is shown in Figure 2.

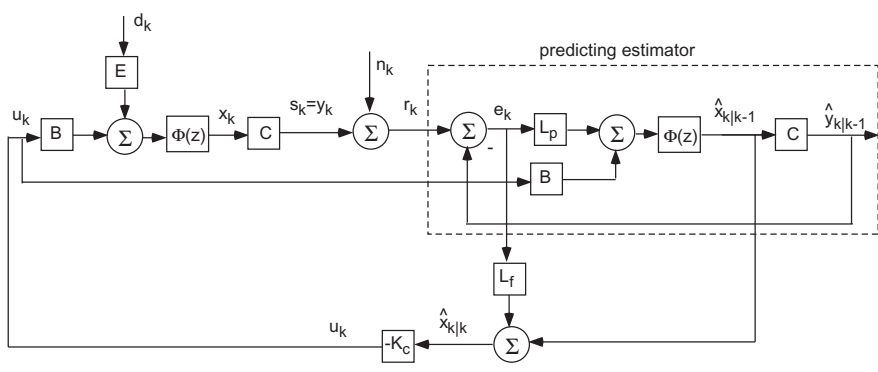

Fig. 2. Feedback system with state feedback based on a filtering estimator. The predicting estimator is in the dashed box.

Under appropriate hypotheses, the solution to the cheap control problem has appealing special properties [6].

Proposition II.2 Consider the cheap control $L Q G$ problem. Assume that $G_{u}$ has no nonminimum phase zeros and relative degree equal to one, and that the control signal is allowed to depend on both current and previous values of the channel output. Then the optimal controller, given by (12)-(13), has the properties that

$$
K_{c}=(C B)^{-1} C A,
$$

and

$$
K(z)=G_{u}^{-1}(z) C \Phi(z) L_{p} .
$$

Furthermore, the sensitivity and complementary sensitivity functions satisfy $S=S_{\text {est }}$ and $T=T_{\text {est }}$, where $S_{\text {est }}=$ $\left(1+C \Phi L_{p}\right)^{-1}$, and $T_{\text {est }}=1-S_{\text {est }}$.

It is easy to verify from Figure 2 that $S_{e s t}$ and $T_{e s t}$ are the sensitivity and complementary sensitivity functions of the feedback loop in a predicting estimator.

\section{Structure of the Optimal Feedback System}

In this section, we use Proposition II.2 to derive interesting properties of the feedback system in Figure 2 and $K(z)$ designed to minimize (4). Recall the output estimation error $\tilde{y}_{k \mid k-1}$ for the predicting estimator, define the associated innovations sequence by

$$
e_{k} \triangleq r_{k}-\hat{y}_{k \mid k-1}=\tilde{y}_{k \mid k-1}+n_{k},
$$

and denote the $z$-transforms of $\tilde{y}_{k \mid k-1}$ and $e_{k}$ by $\tilde{Y}_{p}(z)$ and $E(z)$. Then

$$
\begin{aligned}
\tilde{Y}_{p}(z) & =S_{\text {est }}(z) G_{d}(z) D(z)-T_{\text {est }}(z) N(z), \\
E(z) & =S_{\text {est }}(z) G_{d}(z) D(z)+S_{\text {est }}(z) N(z) .
\end{aligned}
$$

It follows from Proposition II.2 that the channel input and output in Figure 2 are identical to the estimation error and innovations sequence for a predicting estimator, and thus inherit special properties derived from those of the optimal predicting estimator [7].

Proposition III.1 Consider the problem of minimizing (6) in the special case $\lambda=1$. Assume that the hypotheses of Proposition II. 2 are satisfied. Then the optimal control is state feedback (12) with $K_{c}$ given by (14) and $\hat{x}_{k \mid k}$ given by (10). Denote the optimal values of the channel input and output by $s_{k}^{*}$ and $r_{k}^{*}$, respectively. Then

$$
\begin{aligned}
& S^{*}(z)=S_{\text {est }}(z) G_{d}(z) D(z)-T_{\text {est }}(z) N(z), \\
& R^{*}(z)=S_{\text {est }}(z) G_{d}(z) D(z)+S_{\text {est }}(z) N(z) .
\end{aligned}
$$

Furthermore, the channel power constraint must satisfy $P>$ $J_{y}^{*}(1)$, where the optimal cost is equal to the variance of the optimal (predicting) estimation error $J_{y}^{*}(1)=\mathcal{E}^{*}\left\{\tilde{y}_{k \mid k-1}^{2}\right\}$, the optimal channel output $r_{k}^{*}$ is a white noise sequence, and the optimal channel input is orthogonal to the channel output.

We also see that, under optimal control, the feedback system in Figure 2 is equivalent to the communication channel with feedback in Figure 3, in the sense that the responses of the channel input and output to the disturbance and noise in Figure 3 are identical to the responses of the estimation error and innovations sequence to the disturbance and noise in Figure 2.

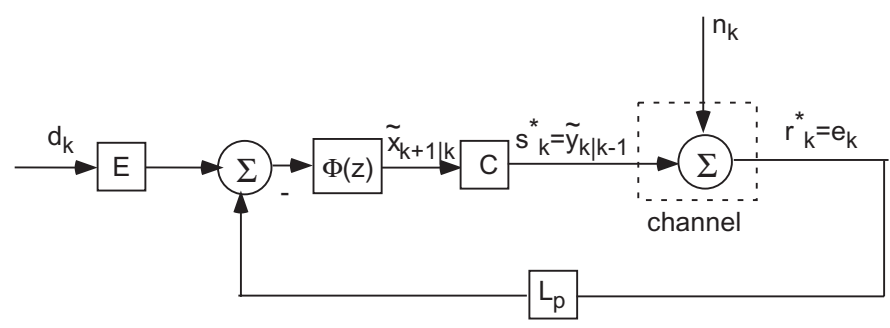

Fig. 3. Under optimal control, the feedback system in Figure 2 is input/output equivalent to a communication channel with feedback.

We have seen that the problem of power limited stabilization with a disturbance over a memoryless Gaussian channel has a solution with the following structure. First, an optimal estimator is applied to obtain the best estimate of the next value of the channel input (which is equal to the plant output) given the previous channel outputs. Second, a control signal is computed that inverts the plant and subtracts this estimate from the plant output. Because the estimate minimizes the mean square estimation error, it follows that the resulting control signal minimizes the variance of the system output, and thus also the power required at the channel input.

\section{ENTROPy RATE AND THE Optimal Estimation ERROR}

We now derive an expression for the optimal prediction estimation error and provide an interpretation in terms of mutual information. The results below are an extension 
of those in [4], wherein the disturbance free stabilization problem was considered.

Given two random variables $a$ and $b$, we denote the mutual information [2] by $I(a ; b)$, and note that $I(a ; b)=$ $h(a)-h(a \mid b)$, where $h(a)$ and $h(a \mid b)$ denote the (differential) entropy of $a$ and the conditional entropy of $a$ given $b$, respectively. The (differential) entropy rate of a stationary, continuous-valued, discrete-time scalar random process $a$ is given by [2] $h_{\infty}(a)=\lim _{k \rightarrow \infty} h\left(a_{k} \mid a^{k-1}\right)$. The entropy rate of a stationary Gaussian random process $a$ may be computed from its power spectral density $\mathcal{S}_{a}(\omega)$ [2] by the formula $h_{\infty}(a)=\frac{1}{2} \log _{e} 2 \pi e+\frac{1}{4 \pi} \int_{-\pi}^{\pi} \log _{e} \mathcal{S}_{a}(\omega) d \omega$. The entropy rate of a random sequence $a$ is related to the problem of estimating $a_{k}$ from previous values $a^{k-1}$. Denote such an estimate by $\hat{a}_{k \mid k-1}$, and the resulting estimation error by $\tilde{a}_{k \mid k-1} \triangleq a_{k}-\hat{a}_{k \mid k-1}$. Then the variance of the minimal mean square estimation error satisfies [2]

$$
\mathcal{E}^{*}\left\{\tilde{a}_{k \mid k-1}^{2}\right\}=(1 / 2 \pi e) e^{2 h_{\infty}\{a\}} .
$$

We now apply the relation between entropy rate and estimation error to the feedback system of Figure 1 with $\lambda=1$. The power spectrum of the channel output may be written as

$$
\mathcal{S}_{r}(\omega)=\left|S\left(e^{j \omega}\right)\right|^{2} \sigma_{n}^{2}\left(1+\left|G_{d}\left(e^{j \omega}\right)\right|^{2} \sigma_{d}^{2} / \sigma_{n}^{2}\right) .
$$

Since $d$ and $n$ are assumed Gaussian, the channel output is also Gaussian, with entropy rate

$$
\begin{aligned}
h_{\infty}(r)= & \frac{1}{2} \log _{e} 2 \pi e \sigma_{n}^{2}+\frac{1}{2 \pi} \int_{-\pi}^{\pi} \log _{e}\left|S\left(e^{j \omega}\right)\right| d \omega \\
& +\frac{1}{4 \pi} \int_{-\pi}^{\pi} \log _{e}\left(1+\left|G_{d}\left(e^{j \omega}\right)\right|^{2} \frac{\sigma_{d}^{2}}{\sigma_{n}^{2}}\right) d \omega .
\end{aligned}
$$

Suppose that the plant $G_{u}$ is strictly proper, has $m$ anti-stable poles $\left|\phi_{i}\right|>1$, and no nonminimum phase zeros. Then it is possible to stabilize the system using a controller with no anti-stable poles, and the sensitivity function must satisfy the Bode integral [8]

$$
\frac{1}{2 \pi} \int_{-\pi}^{\pi} \log _{e}\left|S\left(e^{j \omega}\right)\right| d \omega=\sum_{i=1}^{m} \log _{e}\left|\phi_{i}\right| .
$$

We now provide an interpretation of the third term on the right hand side of (23). Suppose that state feedback $u_{k}=-K_{m e} x_{k}$ is used to stabilize the plant and minimize the energy in the control signal, given by $\sum_{k=0}^{\infty} u_{k}^{2}$. The closed loop transfer function from $d_{k}$ to $r_{k}$ is given by $C \Phi E\left(1+K_{m e} \Phi E\right)^{-1}$. It may be shown from [5, Theorem 6.35 (d)] that $\left(1+K_{m e} \Phi E\right)^{-1}$ is allpass, and thus that the magnitude of the transfer function from $r_{k}$ to $d_{k}$ is identical to that of $G_{d}$. The mutual information rate [9] between the disturbance and channel output satisfies

$$
I_{\infty}(r ; d)=\frac{1}{4 \pi} \int_{-\pi}^{\pi} \log \left(1+\left|G_{d}\left(e^{j \omega}\right)\right|^{2} \frac{\sigma_{d}^{2}}{\sigma_{n}^{2}}\right) d \omega .
$$

Substituting (24) and (25) into (23) and applying the formula (21) shows that the minimum mean square error in estimating $r_{k}$ given $r^{k-1}$ is given by

$$
\mathcal{E}^{*}\left\{\tilde{r}_{k \mid k-1}^{2}\right\}=\sigma_{n}^{2} \prod_{i=1}^{m}\left|\phi_{i}\right|^{2} e^{2 I_{\infty}(r ; d)} .
$$

Let us now relate the problem of estimating the current channel output $r_{k}$ given previous outputs $r^{k-1}$ to that of estimating the current channel input $s_{k}=y_{k}$ given $r^{k-1}$. Denote the estimation errors for $r_{k}$ and $y_{k}$ by $\tilde{r}_{k \mid k-1}$ and $\tilde{y}_{k \mid k-1}$. Then, since $n$ is zero mean and white, it follows that

$$
\mathcal{E}\left\{\tilde{r}_{k \mid k-1}^{2}\right\}=\mathcal{E}\left\{\tilde{y}_{k \mid k-1}^{2}\right\}+\sigma_{n}^{2} .
$$

Combining (27) with (26) yields an expression for the minimal error in estimating the channel input $y_{k}$ given previous values of the channel output $r^{k-1}$ that provides an alternative expression for the minimal power required for stabilization.

Proposition IV.1 Assume that the hypotheses of Proposition II.2 are satisfied and that $\lambda=1$. Then the channel power limit must satisfy the lower bound $P>J_{y}^{*}(1)$, where

$$
J_{y}^{*}(1)=\sigma_{n}^{2}\left(\prod_{i=1}^{m}\left|\phi_{i}\right|^{2} e^{2 I_{\infty}(r ; d)}-1\right) .
$$

As noted in Section III, under the hypotheses of Proposition II.2, the variance of the channel input will be equal to that of the optimal estimation error. The minimal channel capacity required for stabilization is thus

$$
\mathcal{C}>\sum_{i=1}^{m} \log _{e}\left|\phi_{i}\right|+I_{\infty}(r ; d) \quad \text { nats/transmission. }
$$

We now provide an interpretation of the two terms that contribute to channel capacity in (29). First, it follows from [3] that the channel capacity required for stabilization alone is given by $\sum_{i=1}^{m} \log \left|\phi_{i}\right|$. Hence we see that the additional capacity required to stabilize in the presence of a disturbance depends on the mutual information between the disturbance and the channel output, once the plant has been stabilized.

\section{Use of Channel Precompensation, $\lambda \neq 1$.}

If values of $\lambda$ other than one are allowed, then more flexibility is available with which to either achieve smaller variance in the plant output or satisfy a lower channel power requirement. Note that the feedback system of Figure 1 may be rearranged so that the plant has state equations

$$
\begin{aligned}
\bar{x}_{k+1} & =A \bar{x}_{k}+B u_{k}+E \lambda d_{k}, \\
s_{k} & =C \bar{x}_{k} .
\end{aligned}
$$

It follows that changing the parameter $\lambda$ is equivalent to changing the variance of the disturbance input. As a consequence, we may apply the results of Sections III and IV to minimize the power in the channel input simply by replacing $\sigma_{d}^{2}$ with $\lambda^{2} \sigma_{d}^{2}$ in all the respective formulas. 
Consider the problem of minimizing the variance of $s_{k}$ in Figure 1 for a given $\lambda$, and denote the optimal cost by

$$
\left.J_{s}^{*}(\lambda) \triangleq \min _{K} \mathcal{E}\left\{s_{k}^{2}\right\}\right|_{\lambda} .
$$

The value of the cost (6) for the plant output for the controller that achieves the minimum in (32) is equal to

$$
J_{y}(\lambda)=J_{s}^{*}(\lambda) / \lambda^{2} .
$$

Lemma V.1 The variance of the plant output, given by the ratio (33), is a monotonically decreasing function of $\lambda^{2}$.

Proof: It follows by substituting $\lambda^{2} \sigma_{d}^{2}$ for the disturbance variance in (28) that

$$
\frac{J_{s}^{*}(\lambda)}{\lambda^{2}}=\frac{\sigma_{n}^{2}}{\lambda^{2}}\left(\prod_{i=1}^{m}\left|\phi_{i}\right|^{2} e^{2 I_{\infty}(r ; \lambda d)}-1\right) .
$$

Taking the derivative with respect to $\lambda^{2}$ in (34) and simplifying yields the result.

Proposition V.2 Assume that the channel power limit satisfies the lower bound in Proposition II.1, and that the hypotheses of Proposition II.2 are satisfied. Then the variance of the plant output (4) can be made arbitrarily close to the optimal cost $J_{y}^{*}=P / \lambda^{2}$, where $\lambda$ is chosen so that $J_{s}(\lambda)=P$.

Proof: For a given value of $\lambda$, the problem of minimizing $J\left(s_{k}, \lambda\right)$ can be solved by applying Proposition III.1 with $\sigma_{d}^{2}$ replaced by $\lambda^{2} \sigma_{d}^{2}$, and it follows from (28) that

$$
J_{s}^{*}(\lambda)=\sigma_{n}^{2}\left(\prod_{i=1}^{m}\left|\phi_{i}\right|^{2} e^{2 I_{\infty}(r ; \lambda d)}-1\right) .
$$

It is clear by inspection of (35) that $J_{s}^{*}(\lambda)$ is a monotonically increasing function of $\lambda$. Furthermore, as $\lambda \rightarrow 0, J_{s}^{*}(\lambda)$ approaches the limit of Proposition II.1, and as $\lambda \rightarrow \infty$, $J_{s}^{*}(\lambda) \rightarrow \infty$. By continuity, there exists a value of $\lambda$ for which the variance of the channel input is equal to $P$, which is assumed to be greater than the bound from Proposition II.1. The optimal controller $K(z)$ has the form (15), where $L_{p}$ is obtained from the Riccati equation (8) with $\sigma_{d}^{2}$ replaced by $\lambda^{2} \sigma_{d}^{2}$.

It follows immediately from Proposition V.2 and Lemma V.1 that if $P>J_{y}^{*}(1)$, then $\lambda>1$ and $J_{y}^{*}<J_{y}^{*}(1)$. We thus see that if a given channel has a power limit greater than that required in the case $\lambda=1$, then the optimal cost $J_{y}^{*}$ is less than that for $\lambda=1$. Similarly, if the channel has a power limit less than that required in the case $\lambda=1$ (but greater than the limit required for stabilization), then the optimal cost $J_{y}^{*}$ is greater than that for $\lambda=1$.

Example V.3 Consider the system (1)-(2) with

$$
A=\left[\begin{array}{cc}
1.1 & 1 \\
0 & 1.2
\end{array}\right], \quad E=\left[\begin{array}{c}
0 \\
1.5
\end{array}\right], \quad C=\left[\begin{array}{ll}
1 & 1
\end{array}\right],
$$

and transfer function $G_{d}(z)=1.5(z-0.1) /\left(z^{2}-2.3 z+\right.$ $1.32)$. Assume the disturbance and noise have variances $\sigma_{d}^{2}=1$ and $\sigma_{n}^{2}=0.1$, respectively. Plots of $J_{s}^{*}(\lambda)$ and
$J_{s}^{*}(\lambda) / \lambda^{2}$ are depicted in Figure 4. Note that the former is monotonically increasing with $\lambda$ and the latter, as proven in Lemma V.1, is monotonically decreasing; of course these plots intersect for $\lambda=1$. For a given power limit, say $P=10$, one finds the value of $\lambda$ for which $J_{s}^{*}(\lambda)=10$, and then corresponding value of $J_{s}^{*}(\lambda) / \lambda^{2}$ is equal to $J_{y}^{*}$, the optimal disturbance response. For the example $P=10$, these values work out to be $\lambda \approx 1.817$ and $J_{y}^{*} \approx 3.029$.

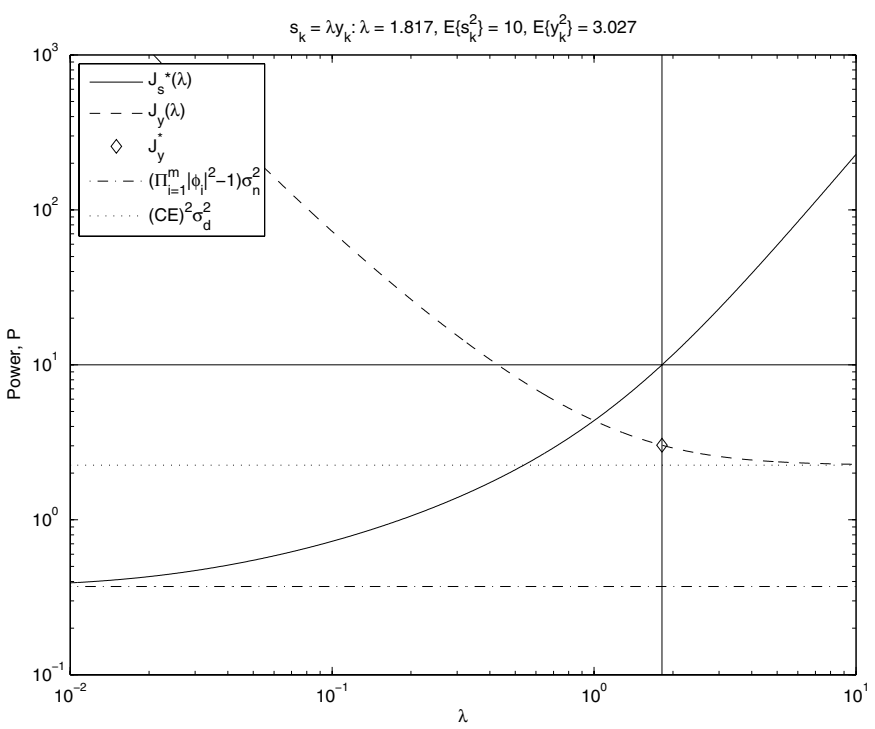

Fig. 4. As $\lambda$ increases, the variance of the channel input increases and that of the plant output decreases.

The next result may be proven by adapting [5, Theorem 6.37].

Proposition V.4 Assume that $G_{d}$ is minimum phase and has relative degree equal to one. Then, in the limit as $\lambda \rightarrow \infty$, $J_{s}(\lambda) / \lambda^{2} \rightarrow \sigma_{d}^{2}(C E)^{2}$.

\section{Optimality OF Linear COMMUNiCATION AND Control: The Scalar Case}

In this section we assume that the plant (1)-(2) is first order $(n=1)$. We also suppose that the channel input and control signal are the outputs of nonlinear, time-varying, and dynamical systems:

$$
s_{k}=f_{k}\left(y^{k}, s^{k-1}\right), \quad u_{k}=g_{k}\left(r^{k}\right) .
$$

We derive a lower bound on the disturbance attenuation achievable with the general communication and control scheme (36), and show that this lower bound is obtained using the linear compensation scheme of Figure 1.

Our first result is applicable to plants of arbitrary order; the remainder to plants of order one only.

Proposition VI.1 Consider the linear system (1)-(2), channel (3), and the general communication and control scheme described by (36). Then

$$
\mathcal{E}\left\{\tilde{y}_{k \mid k}^{2}\right\} \geq \frac{\sigma_{n}^{2}}{\sigma_{n}^{2}+P} \mathcal{E}\left\{\tilde{y}_{k \mid k-1}^{2}\right\} .
$$


Proof: We first show that

$$
I\left(y_{k} ; \hat{y}_{k \mid k} \mid r^{k-1}\right) \geq \frac{1}{2} \log _{e}\left(\mathcal{E}\left\{\tilde{y}_{k \mid k-1}^{2}\right\} / \mathcal{E}\left\{\tilde{y}_{k \mid k}^{2}\right\}\right) .
$$

To do so, we apply an argument similar to that on [2, p. 345]:

$$
\begin{aligned}
I\left(y_{k} ; \hat{y}_{k \mid k} \mid r^{k-1}\right) \stackrel{(a)}{=} h\left(y_{k} \mid r^{k-1}\right)-h\left(y_{k} \mid \hat{y}_{k \mid k}, r^{k-1}\right) \\
\stackrel{(b)}{=} h\left(\tilde{y}_{k \mid k-1} \mid r^{k-1}\right)-h\left(y_{k} \mid \hat{y}_{k \mid k}, r^{k-1}\right) \\
\stackrel{(c)}{=} \frac{1}{2} \log _{e} 2 \pi e \mathcal{E}\left\{\tilde{y}_{k \mid k-1}^{2}\right\}-h\left(y_{k} \mid \hat{y}_{k \mid k}, r^{k-1}\right) \\
\stackrel{(d)}{=} \frac{1}{2} \log _{e} 2 \pi e \mathcal{E}\left\{\tilde{y}_{k \mid k-1}^{2}\right\}-h\left(\tilde{y}_{k \mid k} \mid \hat{y}_{k \mid k}, r^{k-1}\right) \\
\stackrel{(e)}{\geq} \frac{1}{2} \log _{e} 2 \pi e \mathcal{E}\left\{\tilde{y}_{k \mid k-1}^{2}\right\}-h\left(\tilde{y}_{k \mid k}\right) \\
\stackrel{(f)}{\geq} \frac{1}{2} \log _{e} 2 \pi e \mathcal{E}\left\{\tilde{y}_{k \mid k-1}^{2}\right\}-\frac{1}{2} \log _{e} 2 \pi e \mathcal{E}\left\{\tilde{y}_{k \mid k}^{2}\right\},
\end{aligned}
$$

where $(a)$ follows by definition, $(b)$ follows since $\hat{y}_{k \mid k-1}$ is determined from $r^{k-1},(c)$ follows since $\tilde{y}_{k \mid k-1}$ is Gaussian when conditioned on $r^{k-1},(d)$ follows since $\hat{y}_{k \mid k}$ is given, $(e)$ follows since conditioning reduces entropy, and $(f)$ follows since the normal distribution maximizes the entropy for a given second moment. The result (37) follows by applying the data processing inequality [2], $I\left(y_{k} ; \hat{y}_{k \mid k} \mid r^{k-1}\right) \leq$ $I\left(r_{k} ; s_{k} \mid r^{k-1}\right)$, and the fact that mutual information is bounded above by the channel capacity, $I\left(r_{k} ; s_{k} \mid r^{k-1}\right) \leq$ $\frac{1}{2} \log _{e}\left(1+P / \sigma_{n}^{2}\right)$.

Proposition VI.2 Consider the linear system (1)-(2), channel (3), and the general communication and control scheme described by (36). Suppose further that the plant (1)-(2) is first order, and assume that the power limit satisfies

$$
P>\left(A^{2}-1\right) \sigma_{n}^{2} \text {. }
$$

Then communication and control schemes exists for which $\max _{k} \mathcal{E}\left\{\tilde{y}_{k \mid k-1}^{2}\right\}$ is finite and satisfies the lower bound

$$
\sup _{k} \mathcal{E}\left\{\tilde{y}_{k \mid k-1}^{2}\right\} \geq \frac{\left(1+P / \sigma_{n}^{2}\right) \sigma_{d}^{2} C^{2} E^{2}}{\left(1-A^{2}\right)+P / \sigma_{n}^{2}}
$$

Proof: It follows from (1)-(2) that $y_{k}=C A x_{k-1}+$ $C B u_{k-1}+C E d_{k-1}$. If the sequence of channel outputs $r^{k-1}$ is given, then $u_{k-1}$ is determined, and it follows that $\mathcal{E}\left\{\tilde{y}_{k \mid k-1}^{2}\right\}=\mathcal{E}\left\{\left(C A \tilde{x}_{k-1 \mid k-1}\right)^{2}\right\}+(C E)^{2} \sigma_{d}^{2}$, since $\tilde{x}_{k-1 \mid k-1}$ and $d_{k-1}$ are independent. The assumption of a first order plant implies that

$$
\mathcal{E}\left\{\tilde{y}_{k \mid k-1}^{2}\right\}=A^{2} \mathcal{E}\left\{\tilde{y}_{k-1 \mid k-1}^{2}\right\}+(C E)^{2} \sigma_{d}^{2}
$$

Hence, by (37), we have that

$$
\begin{aligned}
\sup _{k} \mathcal{E}\left\{\tilde{y}_{k \mid k-1}^{2}\right\} & \geq A^{2} \sup _{k} \mathcal{E}\left\{\tilde{y}_{k-1 \mid k-2}^{2}\right\} \frac{\sigma_{n}^{2}}{P+\sigma_{n}^{2}}+(C E)^{2} \sigma_{d}^{2} \\
& =A^{2} \sup _{k} \mathcal{E}\left\{\tilde{y}_{k \mid k-1}^{2}\right\} \frac{\sigma_{n}^{2}}{P+\sigma_{n}^{2}}+(C E)^{2} \sigma_{d}^{2}
\end{aligned}
$$

and rearranging yields (40).

Proposition VI.3 Consider the linear system in Figure 1. Assume that the hypotheses of Proposition II.2 and the bound
(39) are satisfied, and that $\lambda$ and $K(z)$ are chosen as in Proposition V.2. Then $\mathcal{E}\left\{\tilde{y}_{k \mid k}^{2}\right\}=\mathcal{E}\left\{\tilde{y}_{k \mid k-1}^{2}\right\} \sigma_{n}^{2} /\left(P+\sigma_{n}^{2}\right)$.

Proof: For a fixed value of $\lambda$, the optimal controller is found by minimizing $\mathcal{E}\left\{\tilde{s}_{k \mid k-1}^{2}\right\}$, and $\lambda$ is chosen so that $\mathcal{E}^{*}\left\{\tilde{s}_{k \mid k-1}^{2}\right\}=P$. Such a value of $\lambda$ exists because the stabilization bound (39) is assumed to be satisfied, and may be found by replacing $\sigma_{d}^{2}$ with $\lambda^{2} \sigma_{d}^{2}$ in the scalar version of the Riccati equation (8), and multiplying by $C^{2}$ to obtain $C^{2} \Sigma=A^{2} C \Sigma-C^{4} \Sigma^{2} /\left(C^{2} \Sigma+\sigma_{n}^{2}\right)+\lambda^{2} \sigma_{d}^{2} C^{2} E^{2}$. Solving for $\lambda^{2}$ yields $\lambda^{2}=P\left(P+\sigma_{n}^{2}\left(1-A^{2}\right)\right) /\left(\sigma_{d}^{2} C^{2} E^{2}\right)$, which by the assumption (39) is guaranteed to be positive. It follows from (11) that $\mathcal{E}^{*}\left\{\tilde{s}_{k \mid k}^{2}\right\}=\sigma_{n}^{2} P /\left(\sigma_{n}^{2}+P\right)$. The result follows by noting that estimates for $y_{k}$ may be obtained from those for $s_{k}$ by dividing by $\lambda$.

Proposition VI.4 In addition to the hypotheses of Proposition VI.3, assume that the plant is first order, and that the power limit satisfies (39). Then choosing $\lambda$ and $K(z)$ as in Proposition V.2 yields

$$
\mathcal{E}\left\{\tilde{y}_{k \mid k-1}^{2}\right\}=\frac{\left(1+P / \sigma_{n}^{2}\right) \sigma_{d}^{2} C^{2} E^{2}}{\left(1-A^{2}\right)+P / \sigma_{n}^{2}}
$$

Proof: The assumption of stationarity, together with (41) and Proposition VI.3, implies that $\mathcal{E}\left\{\tilde{y}_{k \mid k-1}^{2}\right\}=$ $A^{2} \mathcal{E}\left\{\tilde{y}_{k \mid k-1}^{2}\right\} \sigma_{n}^{2} /\left(\sigma_{n}^{2}+P\right)+(C E)^{2} \sigma_{d}^{2}$, and the result follows by rearranging.

\section{CONCLUSIONS}

We have shown how a simple communication and control scheme may be used to stabilize a plant and minimize the variance of the plant output in the case that the plant is minimum phase, relative degree one, and a filtering estimator is used. Extensions to cases where these assumptions fail to hold remain to be worked out. We also showed that for a first order plant no more general control scheme can achieve a lower variance than our linear scheme. Optimality properties for higher order plants remain to be explored.

\section{REFERENCES}

[1] P. Antsaklis and J. Baillieul. Special issue on networked control systems. IEEE Transactions on Automatic Control, 49(9), September 2004.

[2] T. M. Cover and J. A. Thomas. Elements of Information Theory. John Wiley and Sons, New York, 1991.

[3] J. H. Braslavsky, R. H. Middleton, and J. S. Freudenberg. Feedback stabilization over signal-to-noise ratio constrained channels. IEEE Transactions on Automatic Control, 52(8):1391-1403, August 2007.

[4] J. S. Freudenberg, R. H. Middleton, and V. Solo. The minimal signalto-noise ratio required to stabilize over a noisy channel. In Proceedings of the 2006 American Control Conference, pages 650-655, June 2006.

[5] H. Kwakernaak and R. Sivan. Linear Optimal Control Systems. WileyInterscience, 1972.

[6] J. M. Maciejowski. Asymptotic recovery for discrete-time systems. IEEE Transactions on Automatic Control, AC-30(6):602-605, June 1985 .

[7] B. D. O. Anderson and J. B. Moore. Optimal Filtering. Dover, Mineola, NY, 2005.

[8] M. M. Seron, J. H. Braslavsky, and G. C. Goodwin. Fundamental Limitations in Filtering and Control. Springer, 1997.

[9] N. C. Martins and M. A. Dahleh. Feedback control in the presence of noisy channels: "Bode-like" fundamental limitations of performance. IEEE Transactions on Automatic Control, to appear, 2007. 